\title{
The Pioneers of the Mittag-Leffler Functions in Dielectrical and Mechanical Relaxation Processes
}

\author{
FRANCESCO MAINARDI \\ University Bologna and INFN \\ Department of Physics and Astronomy \\ Bologna, ITALY
}

\author{
ARMANDO CONSIGLIO \\ Universität Würzburg \\ Institut für Theoretische Physik und Astrophysik \\ Sanderring 2, 97070 Würzburg, GERMANY
}

\begin{abstract}
We start with a short survey of the basic properties of the Mittag-Leffler functions Then we focus on the key role of these functions to explain the after-effects and relaxation phenomena occurring in dielectrics and in viscoelastic bodies. For this purpose we recall the main aspects that were formerly discussed by two pioneers in the years 1930's-1940's whom we have identified with Harold T. Davis and Bernhard Gross . Key-Words: Mittag-Leffler functions, Laplace transforms, Dielectrics, Electrical Circuits, Viscoelasticty, Aftereffect, Relaxation.
\end{abstract}

Received: September 23, 2019. Revised: April 12, 2020. Accepted: May 14, 2020. Published: June 6, 2020.

\section{Introduction}

The functions of the Mittag-Leffler type are known to play a very important role in the applications of Fractional Calculus, so the original Mittag-Leffler function is recalled as the Queen function of the Fractional Calculus as it was suggested by Mainardi \& Gorenflo in 2007 [1].

The Fractional Calculus is the mathematical theory of the generalized calculus including operators interpreted as integrals and derivatives of non integer order. It has a long history starting from Leibniz and involving eminent mathematicians like Abel, Euler, Fourier, Weyl, Liouville, Riemann just to cite the most prominent ones of the 17-th, 18-th, 19-th centuries. The interested reader can consult any book on Fractional Calculus published after 1974, including those (in order of publication date up to 2010) by: Oldhan \& Spanier [2], Ross [3], Samko, Kilbas \& Marichev [4], Podlubny [5], Hilfer [6], West, Bologna \& Grigolini [7], Kilbas, Srivastava \& Trujillo [8], Diethelm [9], Mainardi [18]. We have restricted our list of books published by 2010 because the list up to nowadays is too long, say impossible: any reader interested to Fractional Calculus can consult the modern books that appear more suitable to his research field.

On the other side the Mittag-Leffler function has a less older story having been introduced by the Swedish mathematician Mittag-Leffler at the beginning of the 20-th century.

For more details the reader is referred to the treatise by Gorenflo et al [11] published in 2014 in the first edition. The second revised and enlarged edition is planned by this year 2020 .

For a minor acquaintance with this function the reader is referred to any book on fractional calculus for the great relevance that the Mittag-Leffler function has in this field.

This paper is organized as follows. In Section 2, we recall some of the basic properties of the MittagLeffler function that is an entire functions in the complex plane. In particular, restricting out attention in the non-negative time domain, we recall for this function its completely-monotonicity properties, the corresponding Laplace transforms and the asymptotic expressions for small and large times. In such a domain we show some plots in order to get a visualization of the Mittag-Leffler function.

In Section 3, we devote our attention to the contributions of the mathematician Harold T. Davis who in the 1920's and 1930's recognized the role of the Fractional Calculus with respect to the Volterra integral equation. Then, as far as we know, he was the first to recognize the Mittag-Leffler function in a contribution by Kennet S. Cole on nerve conduction.

In Section 4, we outline the work of Bernhard Gross who recognized the role of the Mittag-Leffler function in after-effect processes in electrical circuits in 1930's and 1940's. Then, he devoted his attention to the mathematical structure of linear viscoelastic bodies taking profit of the electrical mechanical analogy. Finally, in Section 4, we provide some concluding remarks in order to point out the long-standing story of the applications of the Mittag-Leffler functions due to their scarce popularity in earlier times in applied sciences. 


\section{The Mittag-Leffler functions}

The Mittag-Leffler function is defined by the following power series, convergent in the whole complex plane,

$$
E_{\alpha}(z):=\sum_{n=0}^{\infty} \frac{z^{n}}{\Gamma(\alpha n+1)}, \alpha>0, z \in \mathbb{C} .
$$

We recognize that it is an entire function providing a simple generalization of the exponential function to which it reduces for $\alpha=1$. We also note that for the convergence of the power series in (2.1) the parameter $\alpha$ may be complex provided that $\Re(\alpha)>0$. Furthermore the function turns out to be entire of order $1 / \Re(\alpha)$. The most interesting properties of the Mittag-Leffler function are associated with its asymptotic expansions as $z \rightarrow \infty$ in various sectors of the complex plane. For detailed asymptotic analysis, which includes the smooth transition across the Stokes lines, the interested reader is referred to Paris [14], Wong and Zhao [15].

In this paper we limit ourselves to the Mittag-Leffler function of order $\alpha \in(0,1)$ on the negative real semiaxis where is known to be completely monotone (CM) due a classical result of 1948 by Pollard [12], see also Feller [13].

Let us recall that a function $\phi(t)$ with $t \in \mathbb{R}^{+}$is called a completely monotone (CM) function if it is non-negative, of class $C^{\infty}$, and $(-1)^{n} \phi^{(n)}(t) \geq 0$ for all $n \in \mathbb{N}$. Then a function $\psi(t)$ with $t \in \mathbb{R}^{+}$ is called a Bernstein function if it is non-negative, of class $C^{\infty}$, with a CM first derivative. These functions play fundamental roles in linear hereditary mechanics to represent relaxation and creep processes, see e.g. Mainardi's book [18]. For mathematical details we refer the interested reader to the survey paper by Miller and Samko [16] and to the more recent and exhaustive book by Schilling et al. [17].

In particular we are interested for $t \geq 0$ to the function

$$
e_{\alpha}(t):=E_{\alpha}\left(-t^{\alpha}\right)=\sum_{n=0}^{\infty}(-1)^{n} \frac{t^{\alpha n}}{\Gamma(\alpha n+1)},
$$

with $0<\alpha \leq 1$ that provides the solution to the fractional relaxation equation, see the 1997 survey paper by Gorenflo and Mainardi (1997), see also Mainardi and Gorenflo (2007), Mainardi [18].

For readers' convenience let us briefly outline the topic concerning the generalization via fractional calculus of the first-order differential equation governing the phenomenon of (exponential) relaxation. Recalling (in non-dimensional units) the initial value problem

$$
\frac{d u}{d t}=-u(t), t \geq 0, \text { with } u\left(0^{+}\right)=1
$$

whose solution is

$$
u(t)=\exp (-t)
$$

the following two alternatives with $\alpha \in(0,1)$ are offered in the literature:

$$
\begin{aligned}
& \frac{d u}{d t}=-D_{t}^{1-\alpha} u(t), t \geq 0, u\left(0^{+}\right)=1, \\
& { }_{*} D_{t}^{\alpha} u(t)=-u(t), t \geq 0, u\left(0^{+}\right)=1, .
\end{aligned}
$$

where $D_{t}^{1-\alpha}$ and ${ }_{*} D_{t}^{\alpha}$ denote the fractional derivative of order $1-\alpha$ in the Riemann-Liouville sense and the fractional derivative of order $\alpha$ in the Caputo sense, respectively.

For a generic order $\mu \in(0,1)$ and for a sufficiently well-behaved function $f(t)$ with $t \in \mathbb{R}^{+}$the above derivatives are defined as follows, see e.g. Gorenflo and Mainardi(1997), Podlubny (1999),

$$
\begin{gathered}
D_{t}^{\mu} f(t)=\frac{1}{\Gamma(1-\mu)} \frac{d}{d t}\left[\int_{0}^{t} \frac{f(\tau)}{(t-\tau)^{\mu}} d \tau\right], \\
{ }_{*} D_{t}^{\mu} f(t)=\frac{1}{\Gamma(1-\mu)} \int_{0}^{t} \frac{f^{\prime}(\tau)}{(t-\tau)^{\mu}} d \tau .
\end{gathered}
$$

Between the two derivatives we have the relationship

$$
\begin{aligned}
{ }_{*} D_{t}^{\mu} f(t) & =D_{t}^{\mu} f(t)-f\left(0^{+}\right) \frac{t^{-\mu}}{\Gamma(1-\mu)} \\
& =D_{t}^{\mu}\left[f(t)-f\left(0^{+}\right)\right] .
\end{aligned}
$$

Both derivatives in the limit $\mu \rightarrow 1^{-}$reduce to the standard first derivative but for $\mu \rightarrow 0^{+}$we have

$$
D_{t}^{\mu} f(t) \rightarrow f(t),{ }_{*} D_{t}^{\mu} f(t) \rightarrow f(t)-f\left(0^{+}\right),
$$

In analogy to the standard problem (2.3), we solve the problems (2.5a) and (2.5b) with the Laplace transform technique, using the rules pertinent to the corresponding fractional derivatives. The problems (a) and (b) are equivalent since the Laplace transform of the solution in both cases comes out as

$$
\widetilde{u}(s)=\frac{s^{\alpha-1}}{s^{\alpha}+1},
$$

that yields our function

$$
u(t)=e_{\alpha}(t):=E_{\alpha}\left(-t^{\alpha}\right) .
$$

The Laplace transform pair

$$
e_{\alpha}(t) \div \frac{s^{\alpha-1}}{s^{\alpha}+1}, \quad \alpha>0
$$

can be proved by transforming term by term the power series representation of $e_{\alpha}(t)$ in the R.H.S of (2.2). 
Furthermore, by anti-transforming the R.H.S of (2.11) by using the complex Bromwich formula, and taking into account for $0<\alpha<1$ the contribution from branch cut on the negative real semi-axis (the denominator $s^{\alpha}+1$ does nowhere vanish in the cut plane $-\pi \leq \arg s \leq \pi)$, we get, see also Gorenflo and Mainardi (1997),

$$
e_{\alpha}(t)=\int_{0}^{\infty} \mathrm{e}^{-r t} K_{\alpha}(r) d r
$$

where

$$
\begin{aligned}
K_{\alpha}(r) & =\mp \frac{1}{\pi} \operatorname{Im}\left\{\left.\frac{s^{\alpha-1}}{s^{\alpha}+1}\right|_{s=r \mathrm{e}^{ \pm i \pi}}\right\} \\
& =\frac{1}{\pi} \frac{r^{\alpha-1} \sin (\alpha \pi)}{r^{2 \alpha}+2 r^{\alpha} \cos (\alpha \pi)+1} \geq 0 .
\end{aligned}
$$

Since $K_{\alpha}(r)$ is non-negative for all $r$ in the integral, the above formula proves that $e_{\alpha}(t)$ is $\mathrm{CM}$ function in view of the Bernstein theorem. This theorem provides a necessary and sufficient condition for a $\mathrm{CM}$ function as a real Laplace transform of a non-negative measure. However, the CM property of $e_{\alpha}(t)$ can also be seen as a consequence of the result by Pollard because the transformation $x=t^{\alpha}$ is a Bernstein function for $\alpha \in(0,1)$. In fact it is known that a CM function can be obtained by composing a CM with a Bernstein function based on the following theorem: Let $\phi(t)$ be a CM function and let $\psi(t)$ be a Bernstein function, then $\phi[\psi(t)]$ is a CM function.

As a matter of fact, $K_{\alpha}(r)$ provides an interesting spectral representation of $e_{\alpha}(t)$ in frequencies. With the change of variable $\tau=1 / r$ we get the corresponding spectral representation in relaxation times, namely

$$
\begin{aligned}
& e_{\alpha}(t)=\int_{0}^{\infty} \mathrm{e}^{-t / \tau} H_{\alpha}(\tau) d \tau, \\
& H_{\alpha}(\tau)=\tau^{-2} K_{\alpha}(1 / \tau),
\end{aligned}
$$

that can be interpreted as a continuous distributions of elementary (i.e. exponential) relaxation processes. As a consequence we get the identity between the two spectral distributions, that is

$$
H_{\alpha}(\tau)=\frac{1}{\pi} \frac{\tau^{\alpha-1} \sin (\alpha \pi)}{\tau^{2 \alpha}+2 \tau^{\alpha} \cos (\alpha \pi)+1},
$$

a surprising fact pointed out in Linear Viscoelasticity by Mainardi in his 2010 book [18]. This kind of universal/scaling property seems a peculiar one for our Mittag-Leffler function $e_{\alpha}(t)$. In Fig 1 we show $K_{\alpha}(r)$ for some values of the parameter $\alpha$. Of course for $\alpha=1$ the Mittag-Leffler function reduces to the exponential function $\exp (-t)$ and the corresponding spectral distribution is the Dirac delta generalized function centred at $r=1$, namely $\delta(r-1)$.

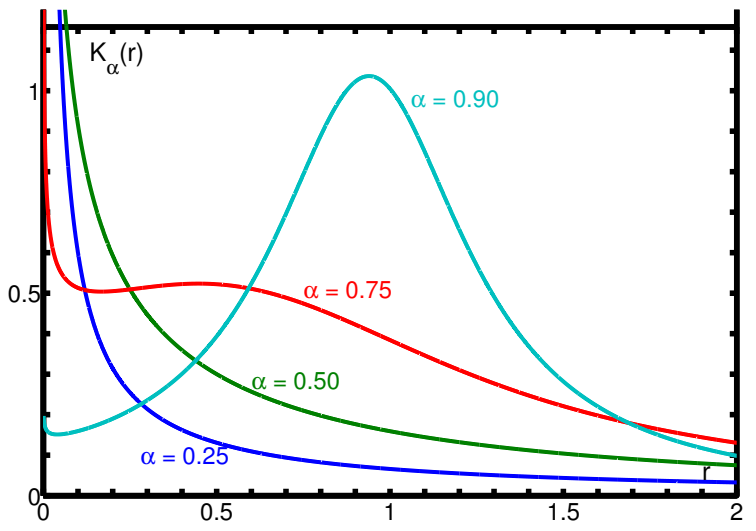

Fig.1 Plots of the spectral function $K_{\alpha}(r)$ for $\alpha=0.25,0.50,0.75,0.90$ in the range $0 \leq r \leq 2$. In Fig 2 we show some plots of $e_{\alpha}(t)$ for some values of the parameter $\alpha$. It is worth to note the different rates of decay of $e_{\alpha}(t)$ for small and large times. In fact the decay is very fast as $t \rightarrow 0^{+}$and very slow as $t \rightarrow+\infty$.

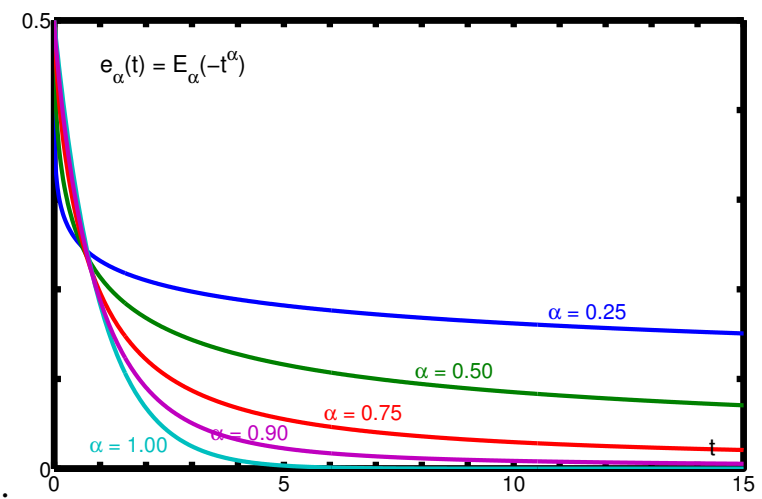

Fig.2 Plots of the Mittag-Leffler function $e_{\alpha}(t)$ for $\alpha=0.25,0.50,0.75,0.90,1$. in the range $0 \leq t \leq 15$

\subsection{The two common asymptotic approxima- tions}

It is common to point out that the function $e_{\alpha}(t)$ matches for $t \rightarrow 0^{+}$with a stretched exponential with an infinite negative derivative, whereas as $t \rightarrow \infty$ with a negative power law. The short time approximation is derived from the convergent power series representation (2.2). In fact for $t \geq 0$,

$$
e_{\alpha}(t)=1-\frac{t^{\alpha}}{\Gamma(1+\alpha)}+\ldots \sim \exp \left[-\frac{t^{\alpha}}{\Gamma(1+\alpha)}\right] . .
$$

The long time approximation is derived from the asymptotic power series representation of $e_{\alpha}(t)$ that turns out to be, see Erdélyi (1955),

$$
e_{\alpha}(t) \sim \sum_{n=1}^{\infty}(-1)^{n-1} \frac{t^{-\alpha n}}{\Gamma(1-\alpha n)}, t \rightarrow \infty,
$$


so that, at the first order,

$$
e_{\alpha}(t) \sim \frac{t^{-\alpha}}{\Gamma(1-\alpha)}, t \rightarrow \infty
$$

As a consequence the function $e_{\alpha}(t)$ interpolates for intermediate time $t$ between the stretched exponential and the negative power law. The stretched exponential models the very fast decay for small time $t$, whereas the asymptotic power law is due to the very slow decay for large time $t$. In fact, we have the two commonly stated asymptotic representations:

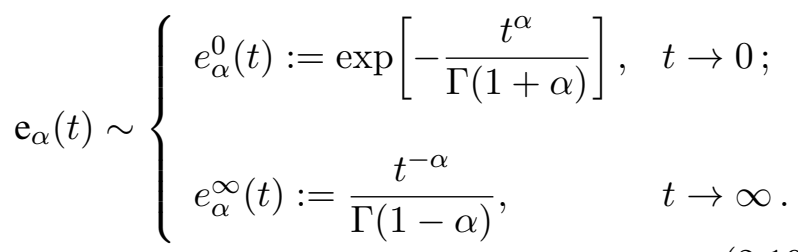

The stretched exponential replaces the rapidly decreasing expression $1-t^{\alpha} / \Gamma(1+\alpha)$ from (3.1). Of course, for sufficiently small and for sufficiently large values of $t$ we have the inequality

$$
e_{\alpha}^{0}(t) \leq e_{\alpha}^{\infty}(t), \quad 0<\alpha<1
$$

In Figs 3-7, for $\alpha=0.25,0.5,0.75,0.90,0.99$, we compare in logarithmic scales the function $e_{\alpha}(t)$ (continuous line) and its asymptotic representations, the stretched exponential $e_{\alpha}^{0}(t)$ valid for $t \rightarrow 0$ (dashed line) and the power law $e_{\alpha}^{\infty}(t)$ valid for $t \rightarrow \infty$ (dotted line). We have chosen the time range $10^{-5} \leq t \leq$ $10^{+5}$.

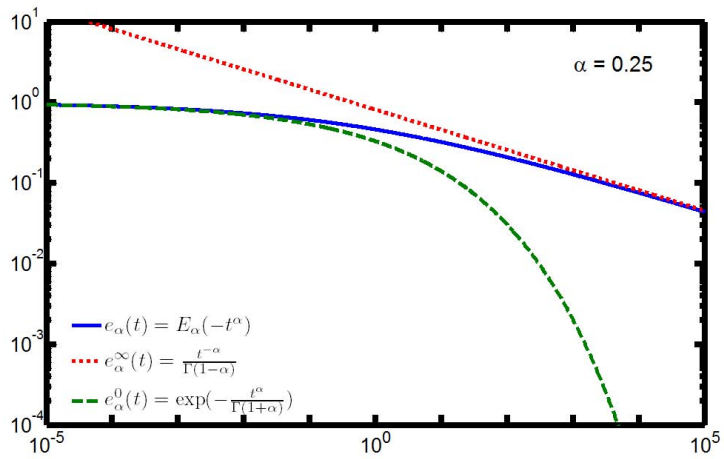

Fig.3 Approximations $e_{\alpha}^{0}(t)$ (dashed line) and $e_{\alpha}^{\infty}(t)$ (dotted line) to $e_{\alpha}(t)$ in $10^{-5} \leq t \leq 10^{+5} ; \alpha=0.25$.

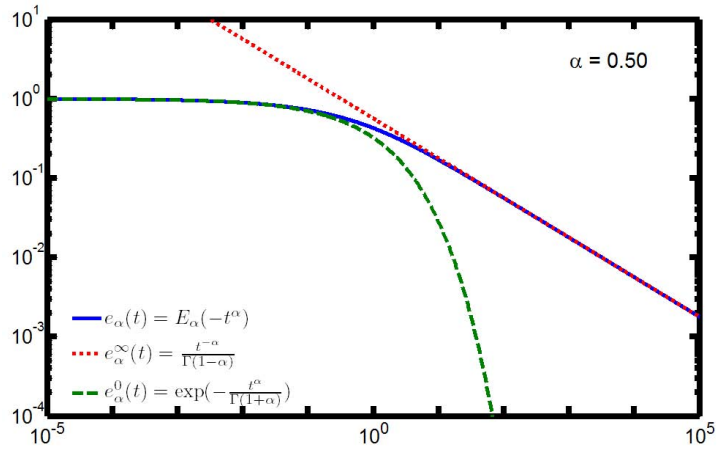

Fig.4 Approximations $e_{\alpha}^{0}(t)$ (dashed line) and $e_{\alpha}^{\infty}(t)$ (dotted line) to $e_{\alpha}(t)$ in $10^{-5} \leq t \leq 10^{+5} ; \alpha=0.50$.

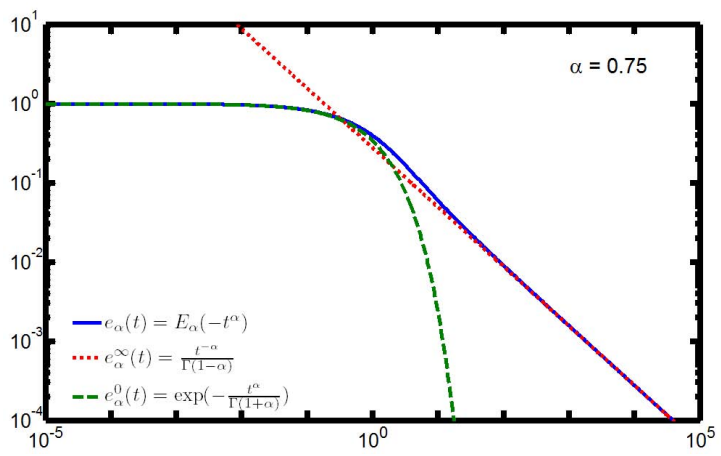

Fig.5 Approximations $e_{\alpha}^{0}(t)$ (dashed line) and $e_{\alpha}^{\infty}(t)$ (dotted line) to $e_{\alpha}(t)$ in $10^{-5} \leq t \leq 10^{+5} ; \alpha=0.75$.

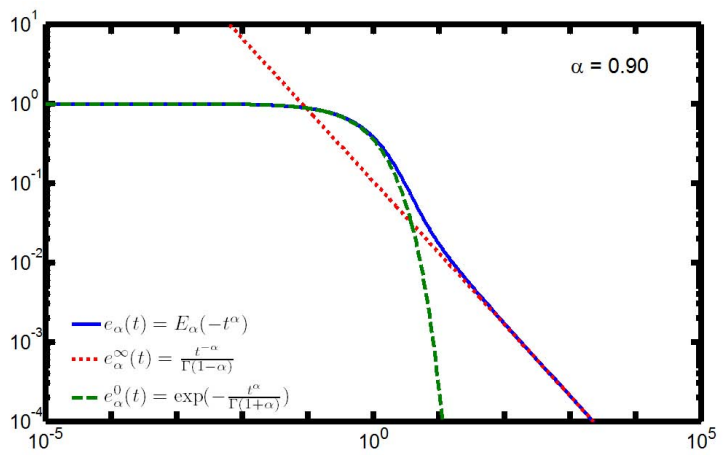

Fig.6 Approximations $e_{\alpha}^{0}(t)$ (dashed line) and $e_{\alpha}^{\infty}(t)$ (dotted line) to $e_{\alpha}(t)$ in $10^{-5} \leq t \leq 10^{+5} ; \alpha=0.90$.

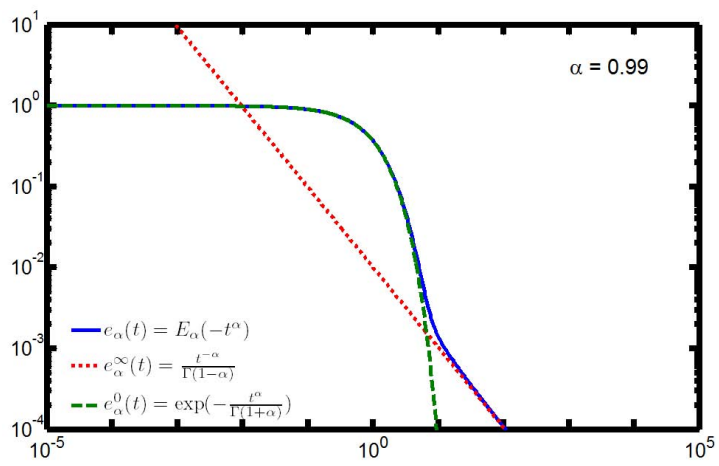

Fig.7 Approximations $e_{\alpha}^{0}(t)$ (dashed line) and $e_{\alpha}^{\infty}(t)$ (dotted line) to $e_{\alpha}(t)$ in $10^{-5} \leq t \leq 10^{+5} ; \alpha=0.99$. 
In the 2014 paper by Mainardi [19] the author, based on numerical computations, has conjectured the relation for $t>0$

$$
f_{\alpha}(t) \leq e_{\alpha}(t) \leq g_{\alpha}(t),
$$

where

$$
\begin{aligned}
f_{\alpha}(t) & :=\frac{1}{1+\frac{t^{\alpha}}{\Gamma(1+\alpha)}} \\
g_{\alpha}(t) & :=\frac{1}{1+t^{\alpha} \Gamma(1-\alpha)} .
\end{aligned}
$$

This conjecture was proved by Simon with rigorous arguments based on the probability theory, see [20].

\section{The contributions of Harold $T$. Davis}

In his 1936 book [21] the mathematician Harold T. Davis devoted 3 sections $(7,8,9)$ in Ch II, pp 64-76 to fractional operators, including fractional integrals and derivatives (in the sense of Riemann-Liouville). Then he devoted one section (7) in Ch VI, pp 280-294 on special applications of the Fractional Calculus mostly involving the Mittag-Leffler function.

For the fundamentals of the fractional calculus we recall the previous notes by Davis published in 1924 [22] and in 1927 [23].

For the applications of the Mittag-Leffler we outline the 1930 fundamental paper by Hille and Tamarkin [24] where the authors solved the Abel integral equations of the second kind by using the Mittag-Leffler function. But this mathematical paper presumably was not observed by Davis who outlined in Ch VI different applications of the Mittag-Leffler functions in some examples. Hereafter we describe the example 4 where Davis reports on two notes by Kennet Cole on nerve conduction published 1933 in the proceedings of the First Symposium on Quantitative Biology (Cold Spring Harbor), see [25]. This report gave to Davis the occasion to interpret the solution of Cole in terms of the Mittag-Leffler function of which Cole (being a physicist interested to biology) was not aware. In our opinion, this result may be considered the first appearance of the Mittag-Leffler functions in applied sciences.

\subsection{The Mittag-Leffler function in nerve conduction}

A living nerve can be stimulated by passing a direct current through a short portion of it between two electrodes, provided the potential difference exceeds a certain critical value known as the rheobase. As the duration of the potential applied across the electrodes decreases, it is found that the intensity necessary for stimulation increases rapidly in a hyperbolic manner. The following analysis is designed to explain this phenomenon.

An idealized nerve fiber consists of a cylindrical core of electrolyte covered with a thin sheath or membrane. It is assumed that a local threshold change of the normal potential difference across the membrane will stimulate the fiber and cause an impulse to be propagated.

The problem is then to express analytically the strength of stimulus which, when applied to the nerve bundle as a whole, will change the potential difference across the membrane of an individual fiber by a threshold amount in a given time

To begin with, experimental evidence points to the conclusion that the electrical behavior of the nerve fiber may be simulated by the type of circuit illustrated in the figure, where $r$ and $R$ are constant resistances and the element $P$, called the polarization element, has an impedance defined by the following equation :

$$
K p^{\alpha} \rightarrow I_{P}(t)=e_{p}(t) \quad 0<\alpha<l .
$$

$I_{r}(t)$ and $I_{P}(t)$ are the instantaneous currents in $r$ and $P$. Then $e_{p}(t)$ is the potential across the element $P$ and $p$ denotes operator $d / d t$. The positive constant $K$ is determined experimentally.

No combination of electrical circuits with ordinary resistances and capacities is known to lead to an impedance of the form postulated, but experimental evidence appears to indicate that such an impedance is essential to the description of the curious electrical behavior of biological materials in general and of nerve fibers in particular.

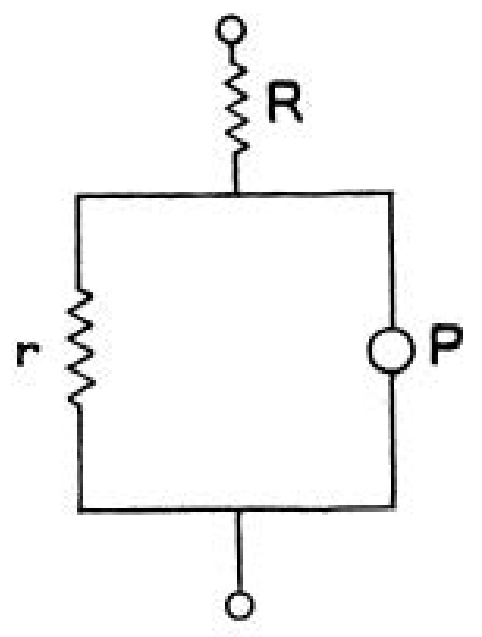

Fig.8 The Cole circuit for nerve conduction 
Referring now to the figure, we compute the relationship between $I_{p}(t)$ and $e_{p}(t)$, when a constant electromotive force (e. m. f.) $E$, is applied across the electrodes.

Applying the Kirkoff laws for the circuits and applying the Laplace transform the solution is easily found to be

$$
e_{P}(t)=\frac{E R}{R+r}\left[1-E_{\alpha}\left(-\lambda t^{\alpha}\right)\right],
$$

where

$$
\lambda=K(R+r) / R r .
$$

Using non dimensional units for the current and the time, we have the following visualization of Eqs (3.2)(3.3) at variance of selected values the parameter $\alpha \in$ $(0,1]$. We note that for $\alpha=1$ the Mittag-Leffler reduces to the exponential function with a great difference with the cases $\alpha \min (0.1)$ because of the strong variability for small times ad low variabilty for large times as expected from the corresponding asymptotic expressions stated in Eq (2.19).

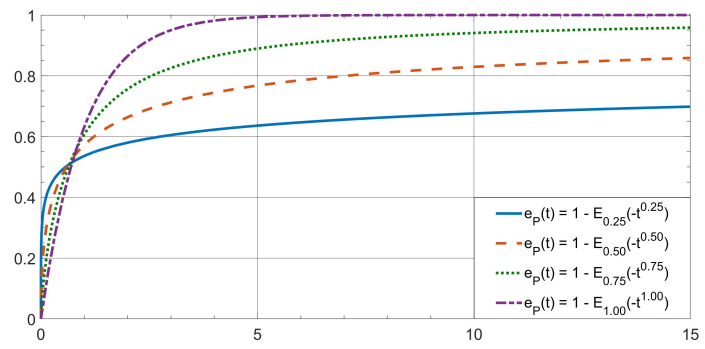

Fig.9 The potential across the element $P$ in the Cole circuit for nerve conduction, versus time, for selected values of $\alpha=0.25,0.50,0.75,1.00$.

We then outline that Kennet Cole with his younger brother Robert published two seminal papers on dielectric relaxation, $[26,27]$ illustrated in the historical survey by Valerio, Machado and Kiryakova [28] on some pioneers of the applications of fractional calculus. Indeed the laws for dielectric relaxation that Cole brothers introduced and known as Cole-Cole models can be considered expressed in terms of fractional operators and Mittag-Leffler function, even if the authors were (once again) not aware of these interpretations. For more details on dielectric laws interpreted by fractional operators and special functions of the MittagLeffler type, we refer the reader to the more recent survey by Garrappa, Mainardi and Maione [29]

\section{The contributions of Bernhard Gross}

Bernhard Gross was a physicist graduated in Stuttgart, Germany. Since he arrived in Rio de Janeiro in Brazil in 1933, for many years he was associated with an electrical engineering department. He became familiar with the mathematics of dielectric phenomena and electrical network theory - the counterparts of viscoelastic effects - a long time before he had his attention directed to the latter. This will perhaps excuse his tendency of bringing into the discussion of viscoelastic theory concepts and methods which originated in electrical theory.

After producing several papers in electrical circuits, Gross gave the opportunity in 1939 to F.M de Oliveira Castro to discuss with more details the mathematical theory of the voltage of a capacitor which has a non exponential after-effects [30]. Indeed Gross in 1937 [31] had noted that the voltage curve of a capacitor which has after-effects, when it is discharged, is not exponential and depends on the charging time; after a temporary short circuit, residual charges appear again. The discussion of Gross needed of a rigorous mathematical approach based on Volterra integral equations that leads to Mittag-Leffler functions.

In the following we report briefly the approach by Castro but we use the most convenient tool of the Laplace transforms.

\subsection{Introduction of the paper by Castro}

In analogy to the simple differential equation of the capacitor without after-effects, an integro-differential equation can be set up if the superposition principle is valid for the capacitor with after-effects. Gross has solved this approximately and the solution reproduced the observed behavior correctly in principle. The degree of approximation could not be overlooked; so the question remained open whether existing deviations between the measurement and the calculation are due to inadequacies of the calculation or to the nonfulfillment of its requirements.

The aim of the present work is a strict integration of the discharge equation. Their implementation provides a satisfactory theory of capacitor discharge; moreover, we believe that it is a contribution to the theory of the Volterra integral equation, which is also of interest from a purely mathematical point of view. The prerequisite for the calculation is the selection of a suitable after-effect function. We have chosen the Schweidler expression $\beta t^{-n}$ for this, which has been shown time and time again to best represent the course of the recharging current in a wide inter- 
val [32, 33, 34, 35, 36]; possible deviations for short times, such as Voglis [36] has determined is of no importance here.

The work is organized as follows: the discharge equation is first converted to the form of a Volterra integral equation by a simple transformation. The general integral of this equation is written and then solved via Laplace Transform technique; the solution can be simplified by limiting it to short discharge times, because here the solving core leads to a known transcendent, the function of Mittag-Leffler [37]. Finally, the solution obtained here is compared with the formulas given by Gross [31]; in the area for which they were derived, they prove to be a suitable approximation.

\subsection{The basic equation of the problem}

The assignments of an imperfect capacitor may be isolated at the instant $t=0$. The voltage curve $U(t)$ is then calculated according to Gross [31] as an integral of the equation:

$$
C \frac{d U}{d t}+\frac{U}{R}+\int_{0}^{t} \frac{d U}{d \tau} \phi(t-\tau) d \tau+i_{0}(t)=0
$$

Here $C$ is the geometric capacitance of the arrangement, $R$ is the ohmic terminal resistance, $\phi$ is the after-effect function and $i_{0}$ is the recharging current that results from all voltage changes during charging. We restrict ourselves to the two particularly important cases of discharging after a charge that took place during the time $t_{0}$ with a voltage $U_{0}$, and recharging which is preceded by a full charge with $U_{0}$, and then by a short circuit during the time $t_{0}$.

Then we can write:

$$
\begin{gathered}
i_{0}(t)=\delta U_{0} \phi\left(t+t_{0}\right) \\
{[U(t)]_{0}=U(0)}
\end{gathered}
$$

For the discharge is:

$$
\delta=1 \quad \text { and } \quad U(0)=U_{0}
$$

and for recharge is:

$$
\delta=-1 \quad \text { and } \quad U(0)=0
$$

Eq. 4.3 represents the initial condition which has to be satisfied.

$\phi(t)$ is set, for the reasons given earlier, as:

$$
\phi(t)=\beta t^{-n}, \quad(0 \leq n \leq 1, \beta>0)
$$

\subsection{Transformation of the basic equation into a Volterra integral equation of the second kind}

Eq. 4.1 is an integro-differential equation for $U(t)$, which can be immediately brought to the well-known form of a Volterra integral equation. It is introduced the unknown:

$$
\psi(t)=\frac{d U}{d t}
$$

obtaining

$$
\psi(t)+\int_{0}^{t} \psi(\tau) K(t-\tau) d \tau=f(t) .
$$

The kernel of this equation is:

$$
K(t-\tau)=\lambda\left[1+k(t-\tau)^{p-1}\right] .
$$

The right term is:

$$
f(t)=-\left[\lambda U(0)+\frac{i_{0}(t)}{C}\right]
$$

The function $f$ is restricted in the interval $0 \leq t \leq a$ ( $a$ finite), when $0<c \leq t_{0}$. In the above equations this abbreviations are used:

$$
k=\beta R, \quad p=1-n, \quad \lambda=1 / R C
$$

In the most general case, the discussion of the form of the solution and its application for performing numerical calculations is cumbersome; let us so first deal with two particularly important cases.

\subsection{Case a: $R=\infty$; very short charging or discharging times}

If the charging and discharging time is very short, experience has shown that the anomalous current component initially outweighs the ohmic component over a considerable time interval. In this interval $U / R$ can be neglected compared to $i_{0}(t)$. Formally, this is done by setting $R=\infty$.

Here we propose a Laplace Transform based method to solve Eq. 4.6. The sign $\div$ is used to indicate the juxtaposition among a function and its Laplace Transform, so that we have:

$$
\begin{aligned}
\psi(t) & \div \mathcal{L}[\psi(t)]=\tilde{\psi}(s) \\
f(t) \div \mathcal{L}[f(t)] & =\tilde{f}(s) \\
K(t) \div \mathcal{L}[K(t)] & =\tilde{K}(s)
\end{aligned}
$$

It is straightforward to obtain, from Eq. 4.6:

$$
\tilde{\psi}(s)+\tilde{\psi}(s) \times \tilde{K}(s)=\tilde{f}(s)
$$


Hence:

$$
\begin{gathered}
\tilde{\psi}(s)[1+\tilde{K}(s)]=\tilde{f}(s) \\
\mathcal{L}\left[\frac{d U}{d t}\right]=\tilde{\psi}(s)=\frac{\tilde{f}(s)}{1+\tilde{K}(s)} \\
s \tilde{U}(s)-U(0)=\frac{1}{1+\tilde{K}(s)} \tilde{f}(s) \\
\tilde{U}(s)=\frac{U(0)}{s}+\frac{1}{s(1+\tilde{K}(s))} \tilde{f}(s)
\end{gathered}
$$

Note that:

$$
\tilde{K}(s)=\frac{\lambda}{s}+\frac{\beta}{C} \frac{\Gamma(p)}{s^{p}}
$$

The latter expression is simplified as $R \rightarrow \infty(\lambda \rightarrow$ 0 ), and we substitute it inside Eq. 4.11. In particular:

$$
\frac{1}{s(1+\tilde{K}(s))}=\frac{s^{p-1}}{s^{p}+\frac{\beta}{C} \Gamma(p)}=\tilde{e}(s)
$$

The inverse Laplace Transform of Eq. 4.13 gives us the Mittag-Leffler function:

$$
\tilde{e}(s) \div \mathcal{L}^{-1}[\tilde{e}(s)]=E_{p}\left[-\frac{\beta}{C} \Gamma(p) t^{p}\right]
$$

Thus, the solution is:

$$
U(t)=U(0)+\int_{0}^{t} E_{p}\left[-\frac{\beta}{C} \Gamma(p)(t-s)^{p}\right] f(s) d s
$$

with:

$$
f(s)=-\frac{i_{0}}{C}
$$

Now the second special case will be dealt, which leads formally to the same expression as the above.

\subsection{Case b: $[\mathrm{U}-\mathrm{U}(0)] / \mathrm{R}=0$; shortly after the opening}

In Eq. 4.1, instead of $U / R$, we write the expression $U / R-U(0) / R+U(0) / R . U(0) / R$ is constant and we can incorporate it in the right member; this then only changes the value of $f(s)$. If we limit ourself to such short times that $\frac{U-U(0)}{R}$ can be overlooked, we formally fall back to case $a$. The solution is again given by Eq. 4.15; the only difference is that now:

$$
f(s)=-\left(\frac{U(0)}{R C}+\frac{i_{0}(s)}{C}\right)
$$

\subsection{Calculation of the Mittag-Leffler function}

For some special values $E_{p}$ leads to simple expressions. Indeed:

$$
\begin{gathered}
E_{1}(-x)=e^{-x}, \\
E_{0.5}(-x)=e^{x^{2}}[1-\Phi(x)],
\end{gathered}
$$

where $\Phi(x)$ is the Gaussian error integral:

$$
\Phi(x)=\frac{2}{\sqrt{\pi}} \int_{0}^{x^{2}} e^{-s^{2}} d s
$$

Furthermore we have:

$$
E_{0}(-x)=\frac{1}{1+x}, \quad \text { for }|x|<1
$$

For $|x|>1$ the function $E$ is undefined. However, the curve $\frac{1}{1+x}$ still seems to represent a limit curve for the function $E_{p}$ if $p \rightarrow 0$; we do not conclude this on the basis of strict proof, but on the basis of numerical agreement, as we will shortly show.

From an experimental point of view small values of $p$, of the order of 0.1 , are of interest. We have therefore calculated the function $E_{0.1}$ in the range of $x$ in question.

For $x \leq 1$ the calculation can be done with the following series:

$E_{p}(x)=1+\frac{x}{\Gamma(p+1)}+\frac{x^{2}}{\Gamma(2 p+1)}+\ldots+\frac{x^{h}}{\Gamma(h p+1)}+\ldots$

For $x>1$, instead, this is very tedious. However, there are asymptotic formulas that are very already convenient for values of $x$ around 2 or more. The derivation should take place elsewhere.

We have, with $p=1 / m$ and $m$ being an even number for $x \gg 1$ :

$$
\begin{aligned}
& E_{p}(-x)=\sum_{\nu=1}^{n-1} \frac{(-1)^{\nu+1}}{\Gamma(1-\nu / m) x^{\nu}} \\
& \left(1-\frac{\nu / m}{x^{m}}+\frac{\nu / m(\nu / m+1)}{x^{2 m}}-\ldots\right),
\end{aligned}
$$

It is easy to see that the formula usually converges very quickly. In general, the term with $x^{m}$ is negligible compared to 1 . Table 1 gives the values calculated in this way.

For the further execution of the calculation, which still requires an integration on $E$, it would be desirable to find a simpler even if only approximately valid representation of the function $E$. 
As Eq. 4.20 is valid for $p=0$, it is natural to try a generalized approach for small values of $p$

$$
E_{p}(-x)=\frac{1}{1+a x}
$$

One can then determine $a$ so that the slope at the origin is correctly represented, and because of $\Gamma(1+p)=$ $p \Gamma(p)$ it follows from Eq. $4.21 a=1 / p \Gamma(p)$, and thus here:

$$
E_{p}(-x)=\frac{1}{1+x / p \Gamma(p)}, \quad p \ll 1
$$

The values calculated in this way can also be found in Table 1. In particular, the calculations are performed for $p=0.1$.

We can see that the match is very good, and it decreases with increasing $x$. Using the Eq. 4.22 the deviation can be estimated.

For $x \rightarrow \infty$ is given by first approximation given by $1 / \Gamma(1-p) x$, while Eq. 4.24 gives the expression $\Gamma(1+p) / x$. The difference is meaningless for very small values of $p$.

\begin{tabular}{|c|c|c|c|}
\hline $\mathbf{x}$ & $\mathbf{E}_{\mathbf{0 . 1}}(-\mathbf{x})$ & $\mathbf{1 / 1}+\mathbf{1 . 0 5 1} \mathbf{x}$ & $\mathbf{E}_{\mathbf{0 . 5}}(-\mathbf{x})$ \\
\hline 0.0 & 1.000 & 1.000 & 1.000 \\
\hline 0.2 & 0.8259 & 0.8264 & 0.8090 \\
\hline 0.4 & 0.7031 & 0.7040 & 0.6708 \\
\hline 0.6 & 0.6118 & 0.6133 & 0.5678 \\
\hline 1.0 & 0.4856 & 0.4876 & 0.4276 \\
\hline 2.0 & 0.3200 & 0.3224 & 0.2655 \\
\hline 4.0 & 0.1901 & 0.1922 & 0.1370 \\
\hline 6.0 & 0.1353 & 0.1369 & 0.0940 \\
\hline 8.0 & 0.1049 & 0.1063 & 0.0650 \\
\hline 10.0 & 0.0857 & 0.0869 & 0.0564 \\
\hline
\end{tabular}

If the function $E$ can be replaced by Eq. 4.24, the calculation is further simplified.

\subsection{Explicit expression of the solution for short discharge times in the limit case of full charge}

We're considering now $p \ll 1$ and the case of discharge after a full charge. Then if we limit ourselves to short discharge times, $U$ is given by Eqs. 4.15, 4.17 and 4.23:

$$
U(t)=U_{0}-\frac{U_{0}}{R C} \int_{0}^{t} \frac{d s}{1+\frac{\beta}{p C} s^{p}}
$$

If $p$ can be represented as $1 / m$, with $m$ being even, the integral in Eq. 4.29 can be evaluated.

With:

$$
A=\frac{\beta}{p C}
$$

the integral is written as:

$$
J=\frac{1}{p A^{m}} \int_{0}^{A t^{p}} \frac{u^{m-1}}{1+u} d u .
$$

By dividing and integrating it finally follows:

$$
\begin{aligned}
J=\frac{1}{p A^{m}} & {\left[\frac{A^{m-1} t^{1-p}}{m-1}-\frac{A^{m-2} t^{1-2 p}}{m-2}+\ldots-\ldots\right.} \\
& \left.+A t^{1-(m-1) p}-\ln \left(1+A t^{p}\right)\right]
\end{aligned}
$$

This case is completely solved.

\subsection{Comparison between the rigorous solution and Gross's expressions}

Gross [31] obtained an approximate solution to Eq. 4.1 on the assumption that $d U / d \tau$ changes very slowly compared to $\phi(t-\tau)$, and therefore can be taken out of the integral.

Then, in our notation:

$$
U(t)=U_{0} \exp \frac{1}{U_{0}} \int_{0}^{t} \frac{f(s) d s}{1+\frac{\beta C}{p} s^{p}} .
$$

In the two special cases and for $p \ll 1$, the strict solution could be written in the form:

$$
U(t)=U_{0}+\int_{0}^{t} \frac{f(s) d s}{1+\frac{\beta C}{p}(t-s)^{p}} .
$$

This already takes the form of Eq. 4.19. A first condition for the approximation to be valid is therefore given by the requirement $p \ll 1$, or $n \approx 1$. But that is exactly the assumption.

We now want to compare the solutions more closely. a) In the case of very long loading times and discharge times so short that we can truncate Eq. 4.29 at the linear term, the two equations are identical. This is because here, according to Eq. 4.17, $f(s)=$ $-U_{0} / R C=$ const, and thus in Eq. 4.30 one can replace $(t-s)$ with $s$.

b) For arbitrary charging times and short discharging times, the solutions differ in that instead of $(t-s)^{p}$ the expression $s^{p}$ is used in the approximate solution. However, as long as $p$ is very small and $(t-s)^{p}$ is slowly changing, no significant error will be caused by this.

If $(t-s)$ is replaced by $s$, the sum can be easily evaluated and Eq. 4.29 is obtained. With regard to the approximation, what has been said under b) applies. Castro thus concludes that the Gross solution is generally usable and then has the advantage of great simplicity. The conclusions drawn from it, especially 
about the behavior with very short discharge times, remain strictly. Indeed, later in 1940, Gross [38] outlined the fact that De Oliveira Castro has provided a more rigorous solution of the problem dealt by him in an approximate way in 1937 [31].

\subsection{The advent of the Mittag-Leffler functions in linear viscoelasticity}

As we told at the beginning of this section. Gross devoted his attention to the linear theory of viscoelastity based on the electro-mechanical analogy. This was mostly since mid 1940's to mid 1950's, see [39, 40, 41]. Being aware of the Mittag-Leffler function from the paper [30], Gross noted in his 1947 paper [39] that this function is present in a viscoelasti model both in creep and relaxation (with different characteristic times) and provided its spectral density with the corresponding plots, as it was described in Section 2 and Fig 1.

This fact has inspired Mainardi in his $\mathrm{PhD}$ thesis carried out in the late 1960 at the University of Bologna under the supervision of Prof. Caputo. As a matter of fact Mainardi was able to provide a plot of the function $E_{\alpha}\left(-t^{\alpha}\right)$ for the first time in the literature, as one can see in the 1971 papers by Caputo and Mainardi $[42,43]$. by introducing the so-called fractional Zener model. More precisely in the survey [43], some families of models for viscoelastic bodies were introduced generalizing the classical stress-strain relationship of the mechanical models by replacing ordinary derivatives with derivatives of non integer order. For more details the reader is referred i.e. to Mainardi's book [18] published in 2010 as first edition; a revised and enlarged edition is expected soon.

We finally note that on the late 1960's the only handbook (in English) dealing with Mittag Leffler functions was that of the BATEMAN project [44], and moreover marginally in the chapter devoted to "miscellaneous functions".

\section{Conclusions}

After a short survey of the basic properties of the Mittag-Leffler functions we have shown the key role of these functions in dielectrical and mechanical processes, as outlined formerly by Harold Davis and Bernhard Gross. We have discussed how these two researchers have promoted the Mittag-Leffler functions at their times when these functions were practically unknown in applied sciences. In this survey we have illustrated their contributions so it is worth to recall them as pioneers of the applications of the Mittag-Leffler functions outside mathematics
Acknowledgments: The research activity of both authors has been carried out in the framework of the activities of the National Group of Mathematical Physics (GNFM, INdAM), Italy.

We outline that Section 3 is partly taken from the 1936 book by Davis including fig 8, whereas the solution of Cole's circuit has been obtained by us using the Laplace transform in terms of the MittagLeffler function. Furthermore, most of Section 4 has been adapted from our translation from German to English of the 1939 paper by Castro with the solutions obtained by us using Laplace transforms.

\section{References:}

[1] F. Mainardi and R. Gorenflo (2007). Timefractional derivatives in relaxation processes: a tutorial survey, Fract. Calc. Appl. Anal. 10, 269388. [E-print arXiv:0 701454 ]

[2] K.B. Oldham and J. Spanier . (1974). The Fractional Calculus, Academic Press, New York.

[3] B. Ross (Editor) (1975). Fractional Calculus and its Applications, Springer Verlag, Berlin. [Lecture Notes in Mathematics, Vol. 457]

[4] S.G. Samko, A.A. Kilbas, and O.I. Marichev. (1993). Fractional Integrals and Derivatives, Theory and Applications, Gordon and Breach, Amsterdam. [English translation from the Russian, Nauka i Tekhnika, Minsk, 1987]

[5] I. Podlubny (1999). Fractional Differential Equations, Academic Press, San Diego. [Mathematics in Science and Engineering, Vol. 198]

[6] R. Hilfer (Editor) (2000). Fractional Calculus, Applications in Physics, World Scientific, Singapore.

[7] B. West, M. Bologna and P. Grigolini (2003). Physics of Fractal Operators, Springer, New York.

[8] A.A. Kilbas, H.M. Srivastava and J.J. Trujillo (2006). Theory and Applications of Fractional Differential Equations, Elsevier, Amsterdam. [North-Holland Series on Mathematics Studies No 204]

[9] K Diethelm (2010). The Analysis of Fractional Differential Equations, An Application-Oriented 
Exposition Using Differential Operators of $\mathrm{Ca}-$ puto Type, Springer, Berlin. [Lecture Notes in Mathematics Vol.2004]

[10] F. Mainardi (2010). Fractional Calculus and Waves in Linear Viscoelasticity, An Introduction to Mathematical Models, Imperial College Press, London and World Scientific, Singapore. [2-nd Edition in preparation]

[11] R. Gorenflo, A.A. Kilbas, F. Mainardi and S. Rogosin (2014). Mittag-Leffler Functions. Related Topics and Applications, Springer, Berlin. [2-nd Edition in preparation]

[12] H. Pollard (1948). The completely monotonic character of the Mittag-Leffler function $E_{\alpha}(-x)$, Bull. Amer. Math. Soc. 54, 1115-1116.

[13] W. Feller (1971). An Introduction to Probability Theory and its Applications. Wiley, New York. Ch. 6: pp. 169-176; Ch. 13: pp. 448-454.

[14] R.B. Paris (2002). Exponential asymptotics of the Mittag-Leffler function, Proc R. Soc. London A 458, 3041-3052.

[15] R. Wong and Y.-Q Zhao (2002). Exponential asymptotics of the Mittag-Leffler function, Constructive Approximation 18, 355-385.

[16] K.S. Miller and S.G. Samko (2001). Completely monotonic functions, Integral Transforms and Special Functions 12, 389-402.

[17] R.L. Schilling, R. Song, and Z. Vondraček, (2012). Bernstein Functions. Theory and Applications, De Gruyter, Berlin, 2-nd ed. [1-st ed, (2010)]

[18] F. Mainardi (2010). Fractional Calculus and Waves in Linear Viscoelasticity. Imperial College Press, London and World Scientific, Singapore. [Second edition in preparation]

[19] F. Mainardi (2014). On some properties of the Mittag-Leffler function $E_{\alpha}\left(-t^{\alpha}\right)$, completely monotone for $t>0$ with $0<\alpha<1$, Discret. Contin. Dyn. Syst. -Series B (DCDS-B) 19 No. 7 , 2267-2278. [E-print: arXiv: 1305.0161]

[20] T. Simon (2014). Comparing Frechet and positive stable laws, Electron. J. Probab. 19, 1-25. [E-print arXiv:1310.1888]

[21] H.T. Davis (1936). The Theory of Linear Operators, The Principia Press, Bloomington, Indiana.
[22] H.T. Davis (1924). Fractional operations as applied to a class of Volterra integral equations, Amer.J. Math. 46 No 2, 95-109.

[23] H.T. Davis (1927). The application of fractional operators to functional equations, Amer.J. Math. 49 No 1, 123-142.

[24] E. Hille and J.D. Tamarkin (1930). On the theory of linear integral equations, Ann. Math. 31, 479528.

[25] K.S. Cole (1933). Electrical conductance of biological systems, Electrical excitation in nerves, in Proceedings Symposium on Quantitative Biololgy, Cold Spring Harbor, New York, Vol. 1, pp. 107-116.

[26] K.S. Cole and R.H. Cole, (1941). Dispersion and absorption in dielectrics, I. Alternating current characteristics, J. Chemical Physics 9, 341-349.

[27] K.S. Cole and R.H. Cole (1942). Dispersion and absorption in dielectrics, II. Direct current characteristics, J. Chemical Physics 10, 98-105.

[28] D. Valerio, J.T. Machado, and V. Kiryakova (2014) Some pioneers of the applications of fractional calculus, Fract, Calc. Appl. Anal. 17 No 2, 552-578. DOI: 10.2478/s13540-014.0185-1

[29] R. Garrappa, F. Mainardi and G. Maione (2016). Models of dielectric relaxation based on completely monotone functions, Fract. Calc. Appl. Anal. 19, No 5, 1105-1160. DOI: 10.1515/fca2016-0060 [E-print arXiv: 1611.04028 ]

[30] F.M. De Oliveira Castro (1939). Zur Theorie der dielektrischen Nachwirkung, (On the theory of dielectric after-effects) Zeits. f. Physik A 114 No1-2, 116-126. [In German]

[31] B. Gross (1937). Über die Anomalien der festen Dielektrika (About the anomalies of the solid dielectrics) Zeits. f. Physik A 107 No. 3-4, 217234. [In German]

[32] F. Tank (1915). Über den Zusammenhamg der dielektrischen Effektverluste von Kondensatoren mit den Anomlien der Lodung under der Lettung; (Zürcher Dissertation.) Ann. d. Phys. 453, 307-359, [In German]

[33] W. Gnann, (1930). Über die elektrische Leitfahgkeit von amorphen Quarz (About the electrical conductivity of amorphous quartz) Zeits. $f$. Physik A. 66 No 7-8, 436-452. [In German] 
[34] A. Wikstrom (1935). Some electrical properties of ceresin wax Physics 6 No 3, 86-92.

[35] E. Hippauf and R. Stein (1938). Experimental determination of the equation of the absorption current of a dielectric, Physik. Zeits. 2 No 1, 90XX. [In German]

[36] G. M. Voglis (1938),. "\#Uber die dielektrischen Nachwirkungserscheinungen in festen Nichtleitern (About the dielectric after-effects in solid non-conductors), Zeit.f. Physik A. 109 No 1, 5279. [In German]

[37] G. Mittag-Leffler, Acta Mathematica 29, 101, $1905 ;$ 42, 285, 1918.

[38] B. Gross (1940) On after-effect in solid dielectrics Phys Rev 57, 57-59.

[39] B. Gross, (1947). On creep and relaxation, $J$. Appl. Phys. 18, 212-221.

[40] B. Gross. (1948). On creep and relaxation, II. J. Appl. Phys. 19, 257-264.

[41] B. Gross, (1953). Mathematical Structure of the Theories of Viscoelasticity, Hermann \& C., Paris.

[42] M. Caputo and F. Mainardi (1971a). A new dissipation model based on memory mechanism, Pure and Appl. Geophys. (PAGEOPH) 91, 134147. [Reprinted in Fract. Calc. Appl. Anal. 10 No 3, 309-324 (2007)]

[43] M. Caputo and F. Mainardi (1971b). Linear models of dissipation in anelastic solids, Riv. Nuovo Cimento (Ser. II) 1, 161-198.

[44] A. Erdélyi, W. Magnus, F. Oberhettinger and F. Tricomi, (1955). Higher Transcendental Functions, 3-rd Volume, McGraw-Hill, New York . [Bateman Project].

\section{Creative Commons Attribution License 4.0 (Attribution 4.0 International, CC BY 4.0)}

This article is published under the terms of the Creative Commons Attribution License 4.0 https://creativecommons.org/licenses/by/4.0/deed.en_US 